\title{
Linear heating system for measurement of thermoluminescence using 8031/51 microcontroller
}

\author{
P NEELAMEGAM ${ }^{\dagger}$ and A RAJENDRAN* \\ Research \& PG Department of Applied Physics, Nehru Memorial College, Puthanampatti, Tiruchirappalli 621 007, India \\ ${ }^{\dagger}$ Research \& PG Department of Physics, AVVM Sri Pushpam College, Poondi, Thanjavur 613 503, India
}

MS received 3 February 2003; revised 29 April 2003

\begin{abstract}
A linear heating system is developed using a 8031/51 microcontroller for the measurement of thermoluminescence (TL) in alkali halides and other related compounds. This system also measures the temperature and the amount of light emitted by the sample for TL studies.
\end{abstract}

Keywords. Thermoluminescence; microcontroller; temperature measurement; solid-state controller.

\section{Introduction}

Luminescence is the phenomenon in which a substance absorbs energy in some form or the other and reemits a fraction of it as visible or near visible radiation. Thermoluminescence studies are very relevant in materials science for characterizing certain classes of inorganic halides and other related compounds (Pradip et al 1987). TL technique is used in many fields like radiation dosimetry, archaeology, geology and forensic science and quality control industry. Generally in these experiments a sample is excited by gamma or X-rays or other irradiating sources and then heated at a constant rate while the luminescence intensity is monitored. The theory of TL usually assumes that the sample temperature varies linearly with time, although more general theories have been formulated and calculations made for non-linear heating system. Previous descriptions of apparatus for the measurement of TL have been published elsewhere (Colvin et al 1984; Bickerton et al 1987; Neelamegam et al 1992). In this paper the development of the linear heating system is presented.

\section{System description}

The block diagram of the linear heating system is presented in figure 1 . The system hardware consists of a $k$-type thermocouple, photo multiplier tube (PMT), instrumentation amplifier, 8-bit analog to digital converter $(A / D)$, 8bit digital to analog converter $(D / A)$, solid-state controller, programmable peripheral interface (PPI) and 8031/51 microcontroller. A two-row 16 characters LCD display from Hitachi is interfaced with microcontroller to allow user communications and to display the results. The user

\footnotetext{
*Author for correspondence
}

through the terminal keyboard enters experimental variables and modes of operation into the system.

\section{Hardware system}

\section{$3.18031 / 51$ Microcontroller and other peripherals}

The circuit diagram for ADC 0808 and 8255A Programmable Peripheral Interface is shown in figure 2. 8255A has three programmable ports, port $\mathrm{A}$, port $\mathrm{B}$ and port $\mathrm{C}$, which can be programmed either as an input or output port. The ADC output $D_{7}-D_{0}$ are connected to port A of 8255 . The ADC0808 is a monolithic CMOS device with an 8 channels 8-bit analog to digital converter. IC 0808 offers high speed, high accuracy and repeatability. The 8-bit $A / D$ converter uses successive approximation, which can make 100 conversions per $\mu \mathrm{s}$. The output from the amplifier (temperature) and light intensity are given to the input pins 26 and 27 of ADC 0808. The SOC signal, EOC signal and channel select signals of $A / D$ converter are connected to port $\mathrm{C}$ and $D / A$ converter (DAC1408) input $\left(A_{1}-A_{8}\right)$ is connected to port $\mathrm{B}$ of 8255 as shown in the figure. IC 7414 is used as an oscillator for the clock $(300 \mathrm{kHz})$ to the $A / D$ converter.

The circuit diagram of 8031 microcontroller and its associated peripherals is shown in figure 3 . The 8031 chip has a 16-bit address whose low order lines $\left(A_{0}-A_{7}\right)$ are multiplexed with the data bus as $A D_{0}-A D_{7}$ lines. The address line $A_{0}$ to $A_{7}$ from the IC 74373 is connected to the address lines EPROM (IC2764) and RAM (IC6264). The address line $A_{8}$ to $A_{12}$ of EPROM and RAM are connected to $\mathrm{P} 2 \cdot 0$ to $\mathrm{P} 2 \cdot 4$ (higher order address bus) of microcontroller. The data lines of EPROM and RAM are connected to port 0 of microcontroller. The address lines $A_{15}, A_{14}$ and $A_{13}$ of microcontroller are connected to the input $\mathrm{C}, \mathrm{B}$ and $\mathrm{A}$ of the decoder IC 74138. The address 
decoder 74LS138 is used to select memory devices and peripherals. The pins 17 and 29 are NANDed and then used to select RAM. A crystal oscillator of frequency $12 \mathrm{MHz}$ is connected between 18th and 19th pins of 8031. The RC network is connected to pin 9 (RESET pin), which is used to power on reset.

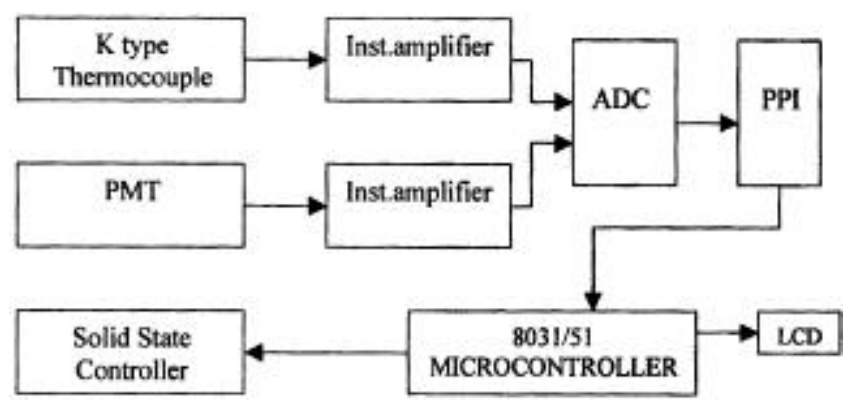

Figure 1. Block diagram of a linear heating system.

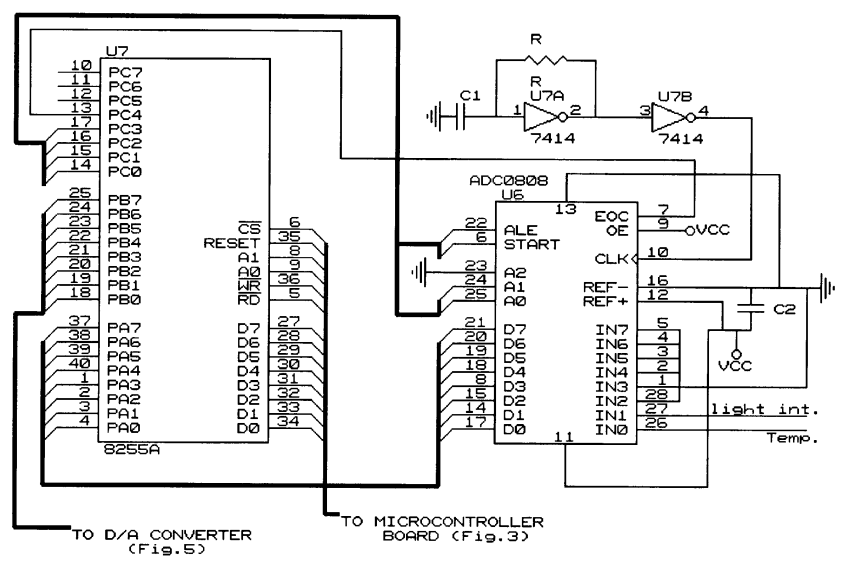

Figure 2. Interface 8255A with ADC 0808.

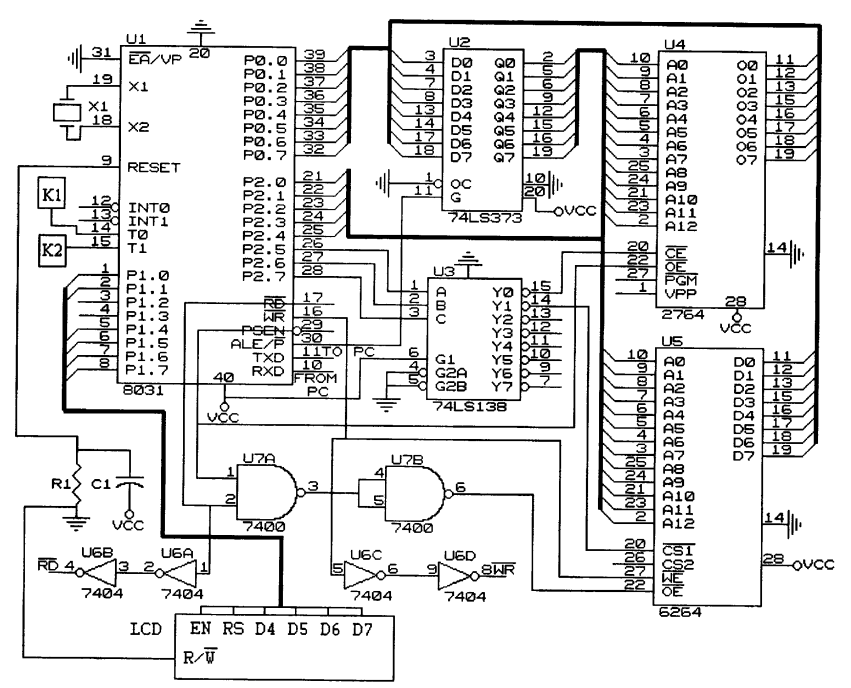

Figure 3. Circuit diagram for 8031/51 microcontroller board.

\subsection{Temperature and light intensity measurement}

In order to investigate the TL, a sample holder (heating element) made of kanthol metal strip of size $3 \times 1.5 \mathrm{~cm}$ is used. The PMT is used as a sensor to measure the amount of light emitted by the sample. The sample holder and photo multiplier tube are kept in a metal box, such that no external light can enter the box. A drawer arrangement is also provided to place the sample in the sample holder. The chromel alumel thermocouple is spot welded on the bottom of the sample holder (kanthol metal strip) to measure the temperature of the sample. A $2 \mathrm{~V}, 40$ amps transformer is used to heat the kanthol strip and hence the sample.

The signal generated by the junction of the thermocouple due to thermal changes was fed to an amplifier circuit specially designed for very low signal amplification as shown in figure 4 . The output signal was amplified to a suitable level by using a instrumentation amplifier read by the microcontroller through $A / D$ converter. A semiconductor temperature sensor AD590 is used to simulate a reference junction and photo multiplier circuit to measure the light intensity, which is emitted from the sample (Neelamegam et al 1992). The microcontroller will read data for temperature and light intensity and it is stored in the memory. Using the keys ( $\mathrm{K} 1 \& \mathrm{~K} 2)$, which is connected to port 3 of microcontroller, data can be retrieved from the memory and it can be displayed. The data also can be sent and stored in the computer (PC) by interfacing a serial interface like ICL232.

\subsection{Solid-state controller}

Figure 5 shows solid-state controller, which is used to control the power to the heater. The method of controlling

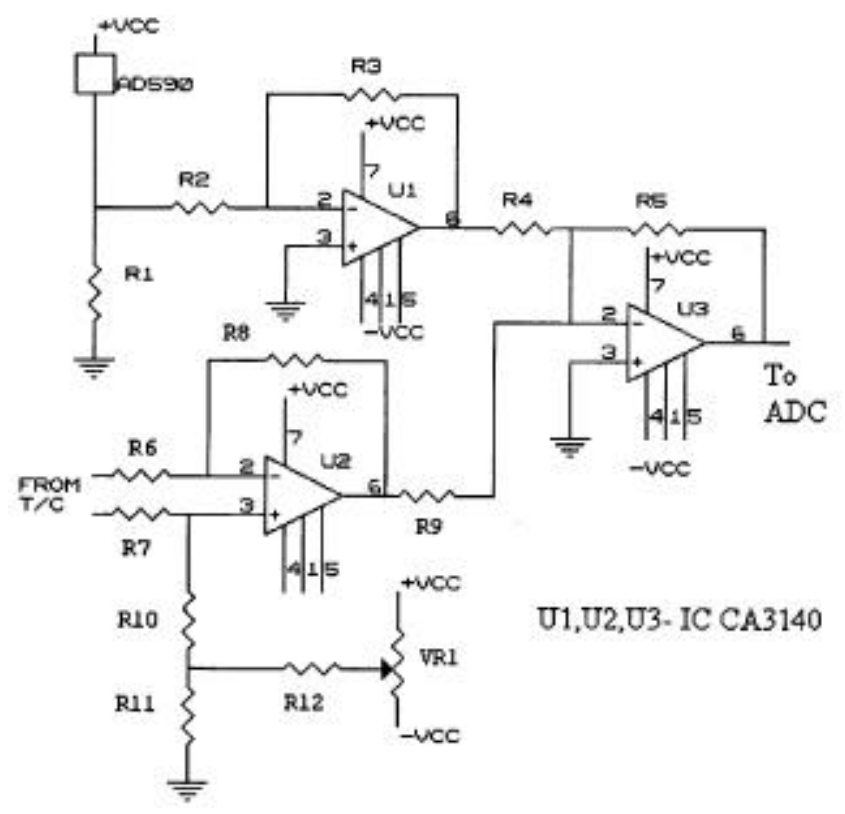

Figure 4. Temperature measurement circuit. 


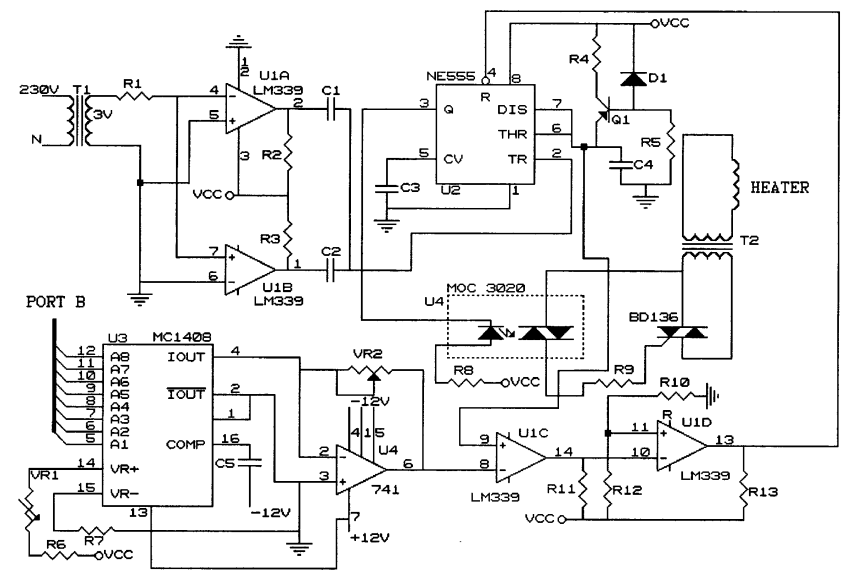

Figure 5. Circuit diagram for solid state controller.

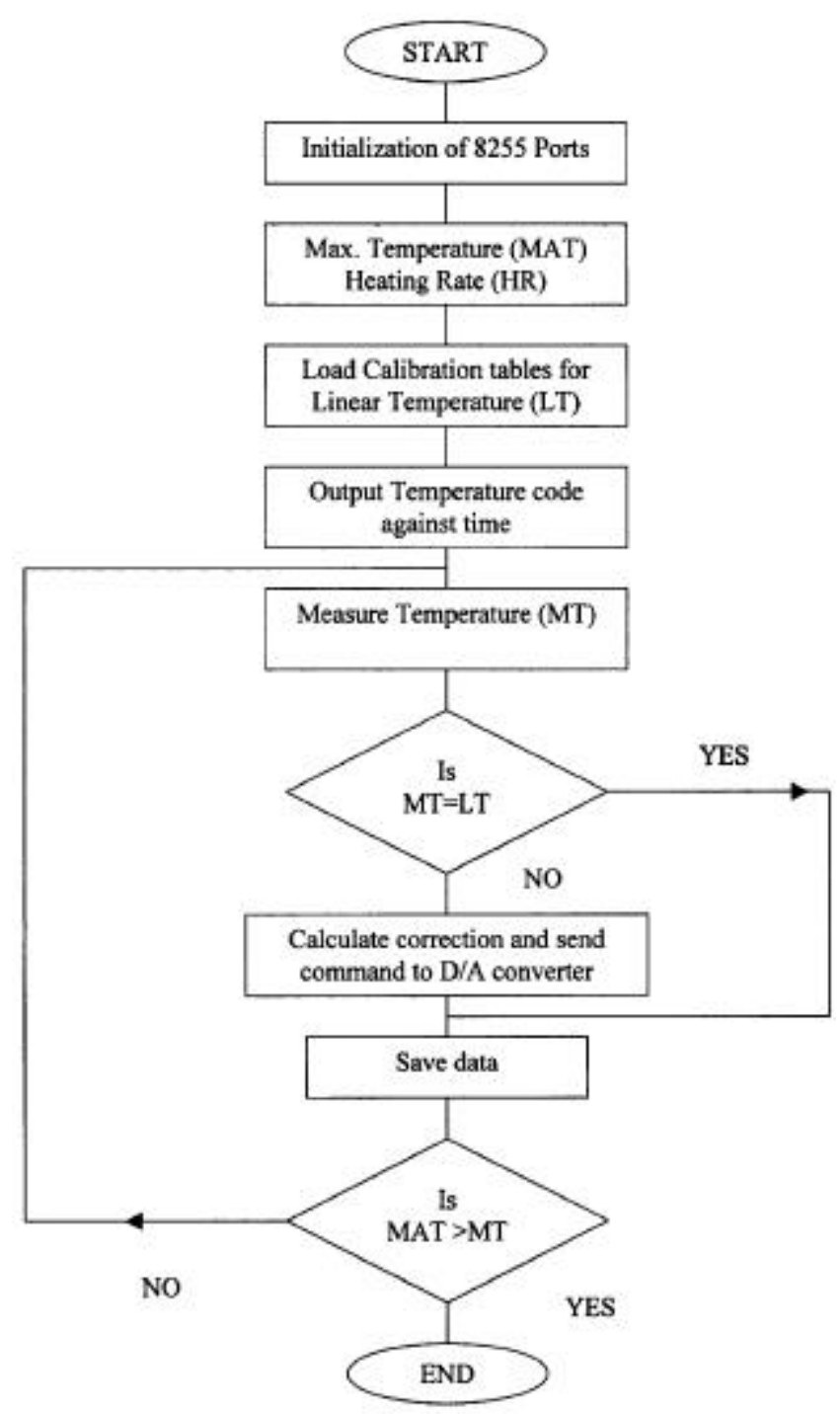

Figure 6. Flowchart for linear heating system. the alternating voltage is to vary the firing angle of a triac (BD136), which is implemented by designing the firing angle control circuitry. A stepped down mains a.c. voltage is fed to two zero crossing detectors (U1A and U1B) whose outputs are connected to the trigger input of IC 555 adopting the monostable multivibrator configuration. The ramp output (pin 6) of 555 is fed to one input of the comparator (U1C) and other input is fed from the output of the D/A converter (DAC1408). Depending upon the $D / A$ converter output voltage the duty cycle of the IC555 is made to vary. This variation in the duty cycle controls the firing angle of the triac by an optically coupled triac driver MOC 3020 circuit and thus the temperature of the heater can be controlled.

\section{Software}

Software is developed in assembly language to initialize 8255A ports, LCD display, selection of temperature or light intensity, to start ADC conversion, to check end of conversion, to read data from ADC, measure the temperature of the sample, light emitted by the sample, to control linear heating, data computation for acquired data, storage of data for temperature and light intensity, switching OFF the heater when the maximum temperature is reached. TL data were collected on each time while the LCD display was updated. Temperature against time and TL against temperature points are stored at the same time. The flowchart for the linear heating system is shown in figure 6 .

\section{Results}

The measurement system was configured to operate over the temperature range of 290 to $573^{\circ} \mathrm{K}$. The linear heating

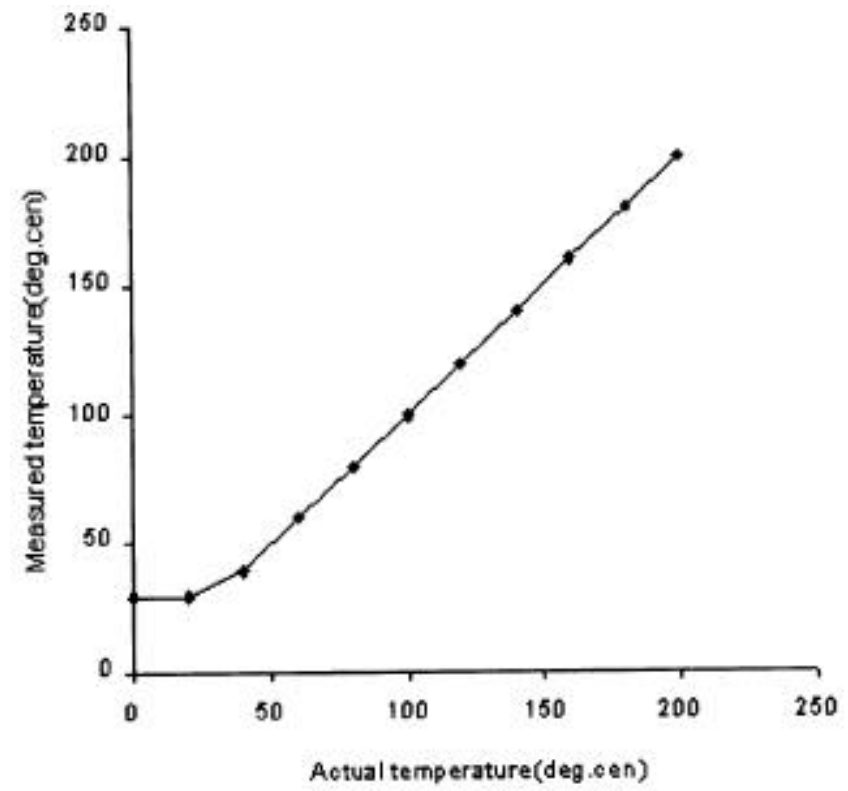

Figure 7. The linear heating of the system. 


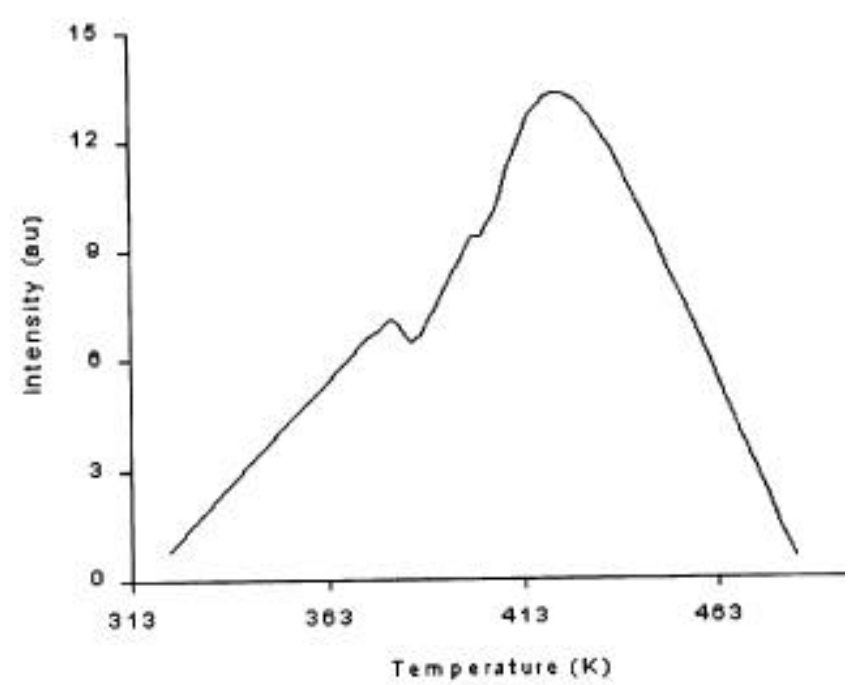

Figure 8. TL glow curves of gamma irradiated Ba doped CsBr.

of the system is tested and shown in figure 7 . The errors are found to be within $0.5 \%$. The linear heating can be varied from 10 to $150 \mathrm{deg} / \mathrm{min}$. The performance of the apparatus has been evaluated using gamma irradiated $\mathrm{Ba}$ doped $\mathrm{CsBr}$ samples. The thermoluminescence glow curves of gamma irradiated $\mathrm{Ba}$ doped $\mathrm{CsBr}$ is shown in figure 8 .

\section{Conclusions}

The microcontroller based linear heating system described has a good performance for thermoluminescence measurements. Greater accuracy for TL measurements would be achieved using 12-bit $A / D$ converter in place of the 8 -bit $A / D$ converter. The use of a microcontroller, the process of temperature control, data acquisition, data storage can automate data computation and presentation. Moreover, the system is easily operated and does not require any programming expertise. The instrument was also tested with different samples to check the reproducibility. The system is highly reliable, less expensive and portable.

\section{References}

Bickerton J C, Green A G J and Viney I V F 1987 J. Phys. E: Sci. Instrum. 20392

Colvin G G, Worpe E A and Gilboy W B 1984 J. Phys. E: Sci. Instrum. 1719

Neelamegam P, Padmanabhan K and Selvasekarapandian S 1992 Meas. Sci. Technol. 3581

Pradip O G, Mazumdar H S and Singhvi A K 1987 Proc. nat. symp. nuclear electronics and instrumentation (Bombay: BARC) 